\title{
Statistics of Carbon Stars in the Galaxy
}

\author{
OMAR M. KURTANIDZE \\ Abastumani Astrophysical Observatory \\ 383762 Abastumani, Republic of Georgia
}

Numerous low-dispersion objective prism spectral surveys have been conducted in recent decades in the yellow-red and near-infrared spectral regions for identification of carbon stars. The data from these spectral surveys have been merged by Stephenson into A General Catalog of Cool Galactic Carbon Stars, containing 5987 entries, the faintest of which are as faint as $I=15.5$. A ten-degree equatorial belt of the Milky Way is now uniformly covered to $I=13.0$. On the basis of these data, characteristics of the surface distribution of carbon stars have been determined. To study the latitude distribution, the whole galactic longitude range was divided into the strips $20^{\circ}$ wide, and then by applying different statistical criteria the uniformity of the C-star distribution was checked. It is shown that in the longitude range $60^{\circ}-120^{\circ}$ most carbon stars are located above the galactic plane, while in the range $260^{\circ}-320^{\circ}$ most are below the plane. In other strips the distribution is uniform, except $40^{\circ}-60^{\circ}$, where a strong concentration of $\mathrm{C}$ stars to the galactic plane is observed. The latitude distribution of carbon stars in the whole galactic longitude range is uniform. To study the longitude distribution, the numbers of $\mathrm{C}$ stars were counted in $5^{\circ}$ bins. The longitude distribution is very non-uniform. A striking increase of the surface density of $\mathrm{C}$ stars is observed away from the galactic center. Their number in the region $310^{\circ}-50^{\circ}$ is two times lower than in the region $130^{\circ}-230^{\circ}$. This could not be fully attributed to galactic absorption, since $\mathrm{M}$ giants show quite the opposite distribution. The surface density of $\mathrm{C}$ stars reaches its maximmum values of $2.3-2.7 \mathrm{deg}^{-2}$ in the CasCyg and Carina directions. The numbers $\mathrm{N}\left(50^{\circ}-130^{\circ}\right)$ and $\mathrm{N}\left(230^{\circ}-310^{\circ}\right)$ are equal to 1281 and 1271 , respectively. It may probably be concluded that most of the carbon stars are located outside the solar circle. By the nearestneighbor method it is shown that the numbers of close associations of carbon stars with each other and with open clusters are not statistically significant. The connection of carbon stars with bright and dark clouds was also studied. 